\title{
Adjusting clinical prediction rules: an academic exercise or the potential for real world clinical applications in perioperative medicine?
}

\author{
Penelope M. A. Brasher, PhD • \\ W. Scott Beattie, MD, PhD
}

Published online: 4 February 2009

(c) Canadian Anesthesiologists' Society 2009

To some, the subject of clinical prediction rules would seem an arcane exercise of limited utility to the everyday clinical anesthesiologist. Nothing could be further from the truth. Clinical prediction rules are plentiful and are in wideranging use in everyday practice. The American Society of Anesthesiologists' physical status classification is one of the most widely used indices of preoperative physical status, and is easy to commit to memory. Thus, it has stood the test of time. Remarkably, it was introduced into everyday practice with little in the way of derivation, validation, or testing, yet it has been proven to perform as well as the Original (Goldman) Cardiac Risk Index, ${ }^{1}$ the Detsky Index, ${ }^{2}$ or the Revised Cardiac Risk Index. ${ }^{3}$

The Revised Cardiac Risk Index (RCRI) was derived from a cohort of 4,315 patients to predict the incidence of major cardiac morbidity in patients over the age of $50 .^{3}$ In the validation set of 1,422 patients, the all-cause mortality doubled, due mostly to increased morbidity in the higher risk population, and the calibration of the index improved significantly. In addition, in the revised index, the coefficients of two risk factors identified from the validation set, diabetes and renal dysfunction, were subsequently found to no longer be significant. These observations highlight just a few of the issues involved with the application of clinical prediction rules. Despite the fact that the RCRI has been

P. M. A. Brasher, PhD (ه)

Centre for Clinical Epidemiology and Evaluation,

VCH Research Institute, University of British Columbia,

828 West 10th Avenue, Vancouver, BC V5Z 1L8, Canada

e-mail: brasher@interchange.ubc.ca

W. S. Beattie, MD, PhD

Department of Anesthesia, University Health Network and the Toronto General Hospital, University of Toronto,

Toronto, ON, Canada utilized in many cohort studies to risk-adjust patients, this index has yet to be externally validated. The performance of the RCRI was reported in a recent meta-analysis of 16 trials reporting on a total of 791,282 patients. ${ }^{4}$ This report found that the RCRI performs poorly (receiver operator characteristic [ROC] values of 0.630 compared to the original validation set value of 0.8 ). There are a number of reasons why the RCRI does not perform as well in "real life", including differences in the frequency of outcomes. In the derivation paper, the mortality was $1 \%$, approximately half of that observed in the largest cohort study utilizing the RCRI, where the mortality rate was found to be $2 \% .^{5}$ Furthermore, there may have been different factors being applied. One factor in the derivation cohort was insulin-treated diabetes, which is now largely regarded as diabetes with or without insulin therapy. The original definition was renal dysfunction, defined as a serum creatinine concentration $>176 \mathrm{mmol} \mathrm{\textrm {L } ^ { - 1 }}$. However, this definition has not been universally applied.

Despite these important shortcomings, i.e., changes in the ROC between derivation and validation, the lack of external validation, or the findings that the RCRI loses accuracy when performed outside the original institution, the RCRI has been incorporated into the ACC/AHA 2007 Guidelines on Perioperative Cardiovascular Evaluation and Care for Non-cardiac Surgery. ${ }^{6}$ Thus, the RCRI has become an integral part of what now constitutes the "standard of care" for patients having non-cardiac surgery. Therefore, by default, any clinician involved in the evaluation of patients prior to non-cardiac surgery should have an understanding of the workings and limitations of this clinical prediction tool. Given the limitations of clinical prediction rules, the statement would also imply that the clinician should know the incidence of important outcomes and have the tools necessary to calibrate the instrument. 
In this issue of the Journal, Janssen et al. present an example of how a simple revision to an apparently "underperforming" prediction rule can lead to good performance. ${ }^{7}$ Readers of the Journal should be aware that these authors have previously published an article detailing the development and external validation of this clinical prediction rule. ${ }^{8}$ As we have alluded to, and as pointed out by these authors, too often when prediction rules perform badly in patient populations, many simply abandon its use or proceed to develop a newer or better rule. ${ }^{9}$ However, "reinventing the wheel" is not an exercise that should be undertaken lightly. The rigorous development of a prediction rule requires large sample sizes, the application of sophisticated statistical methods, and, most importantly, large patient populations for external validation of any newly developed prediction rule. ${ }^{10,11}$ For these reasons, it is important that due consideration be given to the assessment and possible revision of an existing rule.

To assess the performance of an existing model in a new patient population we can graphically compare the observed outcome frequencies in the new data set to the probabilities predicted by the model (Fig. 2 of Janssen et al.). We can also calculate calibration curves by fitting a logistic regression model with the observed outcomes, $Y_{i}$ (event/no event) as the outcome and the linear predictor from the established model, $L P_{i}$ as the only covariate:

$\operatorname{logit}\left(\operatorname{Pr}\left(Y_{i}=1\right)\right)=\alpha+\beta\left(L P_{i}\right)$

In the ideal case of perfect validity, the calibration intercept ( $\alpha$ ) will be 0 and the calibration slope $(\beta)$ will be 1 . If $\beta$ is approximately one, but $\alpha$ differs from zero, this often reflects a difference in the outcome incidence between the patient population on which the model was derived and the new patient population. In such cases, only the intercept of the established model needs to be revised. The article by Janssen et al. provides such an example and illustrates how a correction factor can be calculated and applied to the established model.

In some cases, further revision may be necessary. New regression coefficients for the established model might be based on multiplication of all of the original coefficients by the calibration slope ("logistic recalibration"). More extensive updating methods include re-estimation of regression coefficients of some or all predictors ("model revision") and considering more predictors for inclusion in the model ("model extension"). ${ }^{12}$

As clinicians, we would not consider interpreting the data from an arterial line without first calibrating it. This reasoning can be extended to clinical prediction rules.

As demonstrated by Janssen et al., an existing and reliable model can sometimes be easily adapted for new data by estimating only a single calibration parameter. This will most likely be the case if the covariate distribution in the new patient population is similar to that of the derivation population and if the relative influence of each covariate is correctly specified. We hope the article by Janssen et al. will encourage clinicians and investigators alike to consider calibration of established prediction rules that exist in our everyday practice. The potential implications for improving the allocation of resources and patient outcomes after major surgery are simply too great to ignore.

\section{L'ajustement des règles de prédiction clinique: exercice théorique ou possibilité d'applications cliniques réelles en médecine périopératoire?}

Les règles de prédiction cliniques peuvent sembler à certains un exercice ésotérique d'utilité limitée dans la pratique quotidienne de l'anesthésiologiste clinicien. Pourtant, rien ne pourrait être plus éloigné de la vérité. Les règles de prédiction clinique sont foison et sont utilisées dans toutes sortes de domaines dans la pratique quotidienne. La classification de l'état physique de l'American Society of Anesthesiologists est l'un des indices d'état physique préopératoire les plus couramment utilisés, et il est facile à mémoriser. Ainsi, cette règle a résisté à l'épreuve du temps. Il est à noter que cette classification a été introduite dans la pratique quotidienne avec très peu de modifications, de validation ou d'essai, mais il a été démontré qu'elle était aussi efficace que l'Indice de risque cardiaque original (de Goldman), ${ }^{1}$ l'Indice de Detsky, ${ }^{2}$ ou l'Indice de risque cardiaque modifié. ${ }^{3}$

L'Indice de risque cardiaque modifié (RCRI) a été obtenu à partir d'une cohorte de 4315 patients afin de prédire l'incidence de morbidité cardiaque majeure chez les patients âgés de plus de 50 ans. ${ }^{3}$ Dans le groupe de validation, composé de 1422 patients, la mortalité toutes causes confondues a doublé, principalement en raison de la morbidité accrue dans une population plus à risque, et l'ajustement de l'indice a été significativement amélioré. De plus, dans l'indice modifié, il a été observé que les coefficients de deux facteurs de risque identifiés dans le groupe de validation, nommément le diabète et l'insuffisance rénale, n'étaient plus significatifs. Ces observations mettent en exergue quelques-unes des questions à prendre en considération lors de l'application de règles de prédiction clinique. Malgré le fait que le RCRI a été utilisé dans de nombreuses études de cohorte afin d'ajuster le classement des patients selon le risque, cet indice n'a pas encore été validé de façon externe. L'efficacité du RCRI a été 
rapportée dans une méta-analyse récente portant sur 16 études et incluant un total de 791282 patients. ${ }^{4}$ Ce compterendu a découvert que le RCRI n'est pas très efficace (valeur de la fonction d'efficacité de l'observateur [ROC] de 0,630 comparativement à la valeur de 0,8 pour le groupe de validation originel). Il existe plusieurs raisons pour expliquer l'efficacité moindre du RCRI dans le «monde réel », notamment les différences de fréquence des pronostics. Dans le premier article, la mortalité a atteint $1 \%$, environ la moitié de celle observée dans l'étude de cohorte la plus importante utilisant le RCRI, dans laquelle le taux de mortalité était de $2 \%{ }^{5}$ En outre, différents facteurs pourraient avoir été appliqués. L'un des facteurs dans la première cohorte était le diabète traité à l'insuline, un facteur qui, à l'heure actuelle, est généralement considéré comme le diabète avec ou sans traitement à l'insuline. La définition originale était l'insuffisance rénale, définie comme une concentration sérique de créatinine $>176$ $\mathrm{mmol} / \mathrm{l}$. Toutefois, cette définition n'a pas été appliquée de façon universelle.

En dépit de ces faiblesses importantes, c'est-à-dire, des changements de la courbe ROC entre la description et la validation, le manque de validation externe, ou la découverte que le RCRI perd de sa précision lorsqu'il est réalisé hors de l'institution originale, le RCRI a été intégré aux Directives 2007 d'évaluation cardiovasculaire périopératoire pour les chirurgies non cardiaques de l'ACC/AHA (Guidelines on Perioperative Cardiovascular Evaluation and Care for Non-cardiac Surgery). ${ }^{6}$ Dès lors, par défaut, tout clinicien impliqué dans l'évaluation des patients avant une chirurgie non cardiaque devrait comprendre le fonctionnement et les limites de cet outil de prédiction clinique. Étant donné les limites des règles de prédiction clinique, cette déclaration implique également que le clinicien devrait être conscient de l'incidence de devenirs importants et disposer des outils nécessaires pour ajuster cet outil.

Dans ce numéro du Journal, Janssen et coll. présentent un exemple de comment une modification simple d'une règle de prédiction apparemment « sous-performante » peut en faire un outil performant. ${ }^{7}$ Les lecteurs du Journal devraient toutefois être avertis que ces auteurs ont par le passé publié un article détaillant la mise au point et la validation externe de cette règle de prédiction clinique. ${ }^{8}$ Tout comme nous l'avons laissé entendre et comme ces auteurs l'ont souligné, il arrive trop souvent que, lorsqu'une règle de prédiction n'est pas efficace dans une population de patients, l'utilisation de cette règle est simplement abandonnée par certains; d'autres encore s'attèlent à la tâche de mettre au point une nouvelle règle ou une règle plus performante. ${ }^{9}$ Néanmoins, « la réinvention de la roue » n'est pas un exercice à prendre à la légère. La mise au point rigoureuse d'une règle de prédiction nécessite des tailles d'échantillon importantes, l'application de méthodes statistiques évoluées et, par dessus tout, d'importantes populations de patients pour la validation externe de toute règle de prédiction nouvellement développée. ${ }^{10,11}$ Pour toutes ces raisons, il est important de tenir dûment compte de l'évaluation, voire de la révision, d'une règle de prédiction existante.

Si l'on souhaite évaluer l'efficacité d'un modèle existant dans une nouvelle population de patients, il est possible de comparer graphiquement les fréquences de devenirs observés du nouvel ensemble de données aux probabilités que le modèle prédit (voir Fig. 2, chez Janssen et coll.). Il est également possible de calculer les courbes d'étalonnage en ajustant un modèle de régression logistique aux devenirs observés, $Y_{i}$ (événement/pas d'événement) étant le devenir et l'indice de prédiction linéaire du modèle établi, $L P_{i}$ en tant que seule covariable :

$\operatorname{logit}\left(\operatorname{Pr}\left(Y_{i}=1\right)\right)=\alpha+\beta\left(L P_{i}\right)$

Dans un cas idéal de validité parfaite, le point d'intersection d'étalonnage $(\alpha)$ sera égal à 0 et la pente d'étalonnage ( $\beta)$ sera égale à 1 . Si $\beta$ est environ un, mais $\alpha$ n'est pas égal à zéro, il s'agit souvent d'une différence dans l'incidence des devenirs entre la population de patients de laquelle le modèle est dérivé et la nouvelle population de patients. Dans de tels cas, seul le point d'intersection du modèle établi nécessite une correction. L'article de Janssen et coll. nous fournit l'exemple d'un tel cas et illustre la manière dont un facteur de correction peut être calculé et appliqué au modèle établi. Dans certains cas, des modifications supplémentaires pourraient s'avérer nécessaires. De nouveaux coefficients de régression pour le modèle établi pourraient être calculés en multipliant tous les coefficients originaux par la courbe d'étalonnage (« ré-étalonnage logistique »). Parmi les autres méthodes de mise à jour plus poussées, citons la réévaluation des coefficients de régression de certains ou de tous les indices de prédiction (« révision du modèle ») et la prise en considération d'indices de prédiction supplémentaires afin de les inclure dans le modèle ( « extension du modèle »). ${ }^{12}$

En tant que cliniciens, il ne nous viendrait pas à l'esprit d'interpréter les données d'une canule artérielle sans l'avoir au préalable calibrée. Ce raisonnement peut être appliqué aux règles de prédiction clinique. Comme Janssen et coll. l'ont démontré, un modèle existant et fiable peut parfois être adapté facilement pour traiter de nouvelles données en estimant un seul paramètre d'étalonnage. Il en sera probablement de même dans tous les cas où la distribution des covariables dans la nouvelle population de patients est semblable à celle de la population de départ et si l'influence relative de chaque covariable est spécifiée de façon adéquate. Nous espérons que l'article de Janssen et coll. encouragera les cliniciens et les chercheurs à envisager l'étalonnage de règles de prédiction établies qui sont 
présentes dans notre pratique quotidienne. Les implications potentielles d'une amélioration de la répartition des ressources et des devenirs des patients après une chirurgie majeure sont tout simplement bien trop importantes pour être ignorées.

Conflicts of interest None declared.

\section{References}

1. Goldman L, Caldera DL, Nussbaum SR, et al. Multifactorial index of cardiac risk in noncardiac surgical procedures. N Engl $\mathrm{J}$ Med 1977; 297: 845-50.

2. Detsky AS, Abrams HB, McLaughlin JR, et al. Predicting cardiac complications in patients undergoing noncardiac surgery. J Gen Intern Med 1986; 1: 211-9.

3. Lee TH, Marcantonio ER, Mangione CM, et al. Derivation and prospective validation of a simple index for prediction of cardiac risk of major noncardiac surgery. Circulation 1999; 100: 1043-9.

4. Ford M, Beattie WS, Wijeysundera DN. The revised cardiac risk index for predicting cardiac events and mortality: a systematic review. Anesthesiology 2008; 109: A1420 (abstract).

5. Lindenauer PK, Pekow P, Wang K, Mamidi DK, Gutierrez B, Benjamin EM. Perioperative beta-blocker therapy and mortality after major noncardiac surgery. N Engl J Med 2005; 353: 349-61.
6. Fleisher LA, Beckman JA, Brown KA, et al. ACC/AHA 2007 guidelines on perioperative cardiovascular evaluation and care for noncardiac surgery: executive summary: a report of the American College of Cardiology/American Heart Association Task Force on Practice Guidelines (Writing Committee to Revise the 2002 Guidelines on Perioperative Cardiovascular Evaluation for Noncardiac Surgery). Anesth Analg 2008; 106: 685-712.

7. Janssen KJ, Vergouwe Y, Kalkman CJ, Grobbee DE, Moons KG. A simple method to adjust clinical prediction models to local circumstances. Can J Anesth 2009; 56.

8. Janssen KJ, Kalkman CJ, Grobbee DE, Bonsel GJ, Moons KG, Vergouwe $Y$. The risk of severe postoperative pain: modification and validation of a clinical prediction rule. Anesth Analg 2008; 107: 1330-9.

9. Boersma E, Kertai MD, Schouten $O$, et al. Perioperative cardiovascular mortality in noncardiac surgery: validation of the Lee cardiac risk index. Am J Med 2005; 118: 1134-41.

10. Harrell FE Jr, Lee $L K$, Mark DB. Multivariable prognostic models: issues in developing models, evaluating assumptions and adequacy, and measuring and reducing errors. Stat Med 1996; 15: 361-87.

11. Vergouwe $Y$, Steyerberg EW, Eijkemans MJ, Habbema JD. Substantial effective sample sizes were required for external validation studies of predictive logistic regression models. J Clin Epidemiol 2005; 58: 475-83.

12. Toll DB, Janssen KJ, Vergouwe $Y$, Moons KG. Validation, updating and impact of clinical prediction rules: a review. J Clin Epidemiol 2008; 61: 1085-94. 